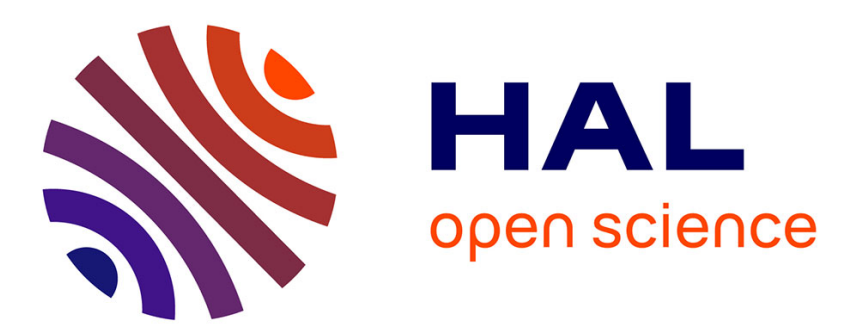

\title{
Simulation of Single Particle Displacement Damage in Si1-xGex alloys - Interaction of Primary Particles with the Material and Generation of the Damage Structure
}

Thomas Jarrin, Antoine Jay, Mélanie Raine, Normand Mousseau, Anne Hémeryck, Nicolas Richard

\section{To cite this version:}

Thomas Jarrin, Antoine Jay, Mélanie Raine, Normand Mousseau, Anne Hémeryck, et al.. Simulation of Single Particle Displacement Damage in Si1-xGex alloys - Interaction of Primary Particles with the Material and Generation of the Damage Structure. IEEE Transactions on Nuclear Science, 2020, 67 (7), pp.1273 - 1283. 10.1109/TNS.2020.2970488 . hal-02469532

\author{
HAL Id: hal-02469532 \\ https://hal.laas.fr/hal-02469532
}

Submitted on 6 Feb 2020

HAL is a multi-disciplinary open access archive for the deposit and dissemination of scientific research documents, whether they are published or not. The documents may come from teaching and research institutions in France or abroad, or from public or private research centers.
L'archive ouverte pluridisciplinaire HAL, est destinée au dépôt et à la diffusion de documents scientifiques de niveau recherche, publiés ou non, émanant des établissements d'enseignement et de recherche français ou étrangers, des laboratoires publics ou privés. 


\title{
Simulation of Single Particle Displacement Damage in $\mathrm{Si}_{1-\mathrm{x}} \mathrm{Ge}_{\mathrm{x}}$ alloys - Interaction of Primary Particles with the Material and Generation of the Damage Structure
}

\author{
Thomas Jarrin, Antoine Jay, Mélanie Raine, Member, IEEE, Normand Mousseau, Anne Hémeryck, \\ Nicolas Richard
}

\begin{abstract}
Primary interaction simulations with neutrons are performed on $\mathrm{Si}_{1-\mathrm{x}} \mathrm{Ge}_{\mathrm{x}}$ alloys with a Monte-Carlo (MC) code using the Binary Collision Approximation (BCA). Then, a statistical study of the collisions cascades development in $\mathrm{Si}_{0.8} \mathrm{Ge}_{0.2}, \mathrm{Si}_{0.7} \mathrm{Ge}_{0.3}$ and $\mathrm{Si}_{0.5} \mathrm{Ge}_{0.5}$ is carried out using Molecular Dynamics (MD) starting from both Si and Ge Primary Knock-On Atoms (PKAs) of $1 \mathrm{keV}, 5 \mathrm{keV}$ and $10 \mathrm{keV}$. The well-known Stillinger-Weber (SW) MD potential which can be employed to study $\mathrm{Si}, \mathrm{Ge}$ and $\mathrm{Si}_{1-\mathrm{x}} \mathbf{G e}_{\mathrm{x}}$ is here coupled to the Ziegler-BiersackLittmark (ZBL) Universal Potential to better describe the collisions between atoms. To account for the stopping power of the electrons the Two-Temperature Model (TTM) is combined with MD. Similar studies are performed on pure $\mathrm{Si}$ and pure $\mathrm{Ge}$ in order to be able to compare our $\mathrm{Si}-\mathrm{Ge}$ alloys damage structures with reference materials. Moreover, data obtained with TTM-MD on $\mathrm{Si}, \mathrm{Ge}$ and $\mathrm{Si}_{1-\mathrm{x}} \mathrm{Ge}_{\mathrm{x}}$ is compared with collision cascades statistical data from $\mathrm{MC}$ codes.
\end{abstract}

\section{INTRODUCTION}

W hile an extensive body of work has been carried out aiming at understanding Displacement Damage (DD) effects in Silicon, the underlying atomistic mechanisms at work in $\mathrm{Si}_{1-\mathrm{x}} \mathrm{Ge}_{\mathrm{x}}$ are still not very well understood. During the 90's the growing interest for the fabrication of Si-Ge devices (same fabrication techniques as the pre-existing ones for $\mathrm{Si}$, tunable band gap, high carrier mobility) motivated many experimental research groups to investigate DD related effects, mainly the behavior of $\mathrm{Si}_{1-\mathrm{x}} \mathrm{Ge}_{\mathrm{x}}$ alloys under ion implantation. The common point they all agree on is the increase in the damage caused by ion implantation with the increase in the alloy's Ge content [1], [2], [3].

This publication is based upon work from COST Action TUMIEE (CA17126), supported by COST (European Cooperation in Science and Technology). The computer simulations were performed using HPC resources from GENCI-CCRT at CEA, DAM, DIF.

T. Jarrin, M. Raine, and N. Richard are with CEA, DAM, DIF, F-91297 Arpajon, France.

N. Mousseau is with Université de Montréal, C.P. 6128, Succursale CentreVille, Montréal, CA-H3C 3J7, Québec, Canada.

A. Hémeryck and A. Jay are with LAAS/CNRS, 7, av. du Col. Roche, F31031 Toulouse, France.
If it is common knowledge that ion implantation or primary particle irradiation creates more defects in Ge than in Si for a given energy and dose, the amount of damage reported for $\mathrm{Si}_{1}$. ${ }_{x} G_{x}$ alloys is far greater than the linear interpolation of the damage created in the two pure compounds [4]. It was also observed that for a $\mathrm{Ge}$ fraction of $50 \%$ or more in the alloy, the amount of damage observed was the same as in pure Ge.

The global simulation approach developed for Silicon in [5], [6] and [7] is here employed to shed light on the atomistic mechanisms responsible for DD in Si-Ge alloys (Fig. 1). The first MC step is dedicated to the interaction between primary particles (at the very origin of DD) with the material and the creation of Primary Knock-On Atoms (PKAs). The PKAs energies and types are used as inputs of the second step (MD) during which the collisions cascades are being propagated in the material. We are using MD combined with the TTM in order to take into account the electronic excitations induced by the moving ions and therefore the energy loss to electronic interactions. Timesteps up to a few ns only can be reached with MD. Therefore, for the damage structures to be further relaxed one needs to employ the $k A R T$ code, an off-lattice kinetic-MC (kMC) code developed to be able to reach long timescales (up to seconds and more). It consists in the third step of the simulation approach. Finally, the representative defects encountered in the damage structures are studied with $a b$ initio techniques to obtain their electronic properties and link experimentally obtained electronic data with the outcomes of the simulation approach. We are especially interested in the behavior of $\mathrm{Si}_{0.8} \mathrm{Ge}_{0.2}$ and $\mathrm{Si}_{0.7} \mathrm{Ge}_{0.3}$ alloys (typical alloy composition used for microelectronic applications) under neutron irradiation at realistic energies. Indeed, the results of the experimental studies presented above could indicate $\mathrm{Si}-\mathrm{Ge}$ devices might be very sensitive to DD. The present paper focuses on the primary particle interaction with the material and the generation of damage structures.

This article presents the first (MC) and second (MD) steps of a global simulation approach to understanding displacement damage in Si-Ge alloys. These first two steps are highlighted by a red box in Fig. 1. 


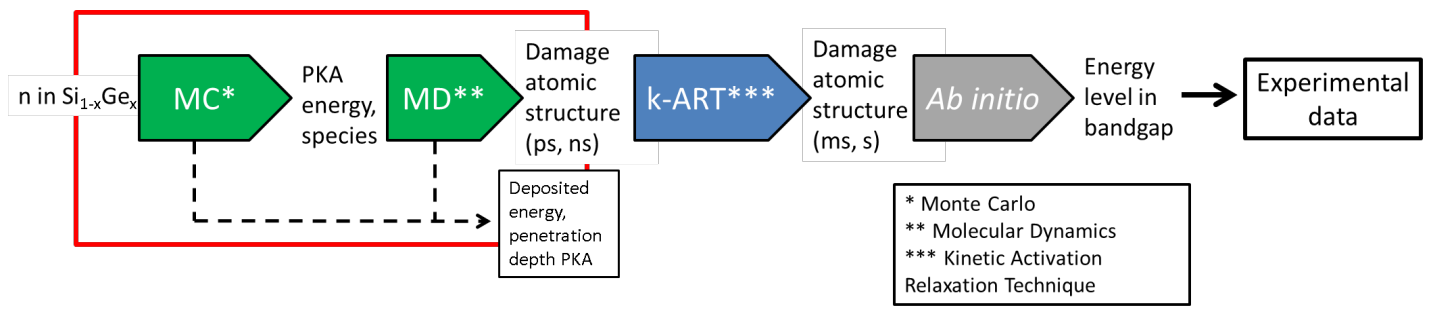

Fig. 1. Scheme of the simulation approach.

\section{Methodology}

\section{A. Monte Carlo}

At the very basis of the DD mechanism is the interaction between incident particles such as neutrons and the target material. When they first shock an atom, incident particles may create fast moving atoms called Primary Knock-On Atoms (PKAs) through non-ionizing energy loss. Therefore, the PKA being the starting point of the collision cascade inside the material, accessing its characteristics is of tremendous importance. In this work, the Monte Carlo Geant4 [11], [12], [13] code in the Binary Collision Approximation (BCA) is employed to simulate the interaction between the primary particle and the target material and thus to obtain PKA energies and types, among other properties. To do so, a very large box of $\mathrm{Si}_{0.8} \mathrm{Ge}_{0.2}$ has been irradiated with neutrons of various energies going from $200 \mathrm{keV}$ to $18 \mathrm{MeV}$. For each neutron incident energy, 1000000 neutrons were fired from the center of the box giving very good statistics. Primary particles interact only once in the simulation box and are killed afterwards (one neutron is forced to create one PKA only). The secondary particles (PKAs in this case) were not tracked. In a second set of simulation focusing on the interaction between the PKAs and the target material we were able to obtain the penetration depth of the PKAs. Both Si and Ge PKAs were used. In Geant4 and other BCA codes matter is considered continuous and materials are not defined by their lattice structure like in MD. Thus, a full and detailed picture of a cascade at the atomic scale (many-body collisions mechanisms, clusterizations of defects and heat spikes) cannot be obtained with this type of MC simulation. Nevertheless, the statistical data which can be obtained with MC codes constitute crucial information for starting a MD simulation. Indeed, we then employ $\mathrm{MC}$ for two purposes: the first $\mathrm{MC}$ simulation step focusing on the creation of PKAs are used as inputs for the next MD step whereas the second set of MC simulations where the PKAs and Secondary Knock-On Atoms (SKAs) are being tracked are used as comparison data for the MD step through common outputs of the two techniques. The Geant4 simulation parameters are the same as those used in [5].

\section{B. Molecular Dynamics}

The purpose of this MD step is to simulate the entire collision cascades, to observe its propagation and more importantly the final damage structure. The many-body interaction potential used for MD techniques provides the possibility to gain precious insights on complex physical processes, unattainable with $\mathrm{MC}$ BCA codes. All the calculations were performed with the LAMMPS code [14].

To simulate the cascade, one atom (the PKA) is being given an initial velocity in the simulation box: this atom will shock the others and create a cascade of collisions. Cascades starting with both $\mathrm{Si}$ and Ge PKAs which were given an initial energy of $1 \mathrm{keV}, 5 \mathrm{keV}$ or $10 \mathrm{keV}$ were simulated. For $1 \mathrm{keV}$ cascades, boxes of 216000 atoms were big enough to contain the cascade extension whereas for $5 \mathrm{keV}$ and $10 \mathrm{keV}$, 1000000 atoms became necessary. Si-Ge alloys being random alloys having a diamond structure just like $\mathrm{Si}$ and $\mathrm{Ge}$, the occupation of diamond lattice sites by $\mathrm{Si}$ or Ge atoms was randomized. Before the PKA is being given its initial velocity, the velocities of the atoms are set so that the overall temperature of the system is $300 \mathrm{~K}$. The influence of the temperature of the system is not being studied here as it would be computationally too expensive to run that many simulations. However it is expected to have an influence on the number of defects at the end of a cascade. The boundaries of the simulation box are made of a thermostat maintained at $300 \mathrm{~K}$. During the simulations the timestep varied from $10^{-5}$ ps to $10^{-3}$ ps over $100 \mathrm{ps}$ (for $1 \mathrm{keV}$ cascades) or $2 \mathrm{~ns}(5 \mathrm{keV}$ and $10 \mathrm{keV}$ cascades). We have used the Two-Temperature Model (TTM) code implemented in LAMMPS to take into account not only the energy lost into the shocks between atoms but also the energy lost to the electrons. In MD simulations, the electrons are not considered explicitly. However, in a TTM-MD simulation, the ionic subsystem can exchange energy with a fictive electronic subsystem through electronic friction and electron-ion (or electron-phonon) interactions via heat transfers [15] [16]. The two subsystems are therefore divided in finite elements, each elements being described by an ionic temperature and an electronic temperature. The TTM assumes that the energy loss can be described by a friction term. Consequently, the usual equations of motions of the atoms are modified to add this term. Due to the consideration of the electron-ion coupling parameter, the subsystem of excited electrons can feed back energy to the ionic system, disturbing the lattice and sometimes melting the material.

This is described by a stochastic force in the equation of motion, which writes itself the following way:

$$
m_{i} \vec{a}_{i}=\vec{F}-\gamma_{i} \vec{v}_{i}+\vec{F}_{\text {stoch }}
$$

$m_{i}$ is the mass of atom i, $\vec{a}_{i}$ its acceleration, $\vec{F}$ the force deriving from the classical potential, $\gamma_{i}$ a friction coefficient, $\vec{v}_{i}$ the velocity of the atom and $\vec{F}_{\text {stoch }}$ the stochastic force 
described above. Depending on the velocity of the particle considered, $\gamma_{i}$ does not have the same expression:

$$
\left\{\begin{array}{l}
\gamma_{i}=\gamma_{p} \quad \text { if } \| \vec{v}_{i}||<v_{0} \\
\gamma_{i}=\gamma_{p}+\gamma_{s} \text { otherwise }
\end{array}\right.
$$

$\gamma_{s}$ is the electronic stopping power, $\gamma_{p}$ the electron-ion coupling parameter and $v_{0}$ the electronic stopping critical velocity. In the TTM, the ionizing stopping power of the PKAs is therefore described by $\gamma_{s}$ and $\gamma_{p}$, whereas in SRIM for example the coupling between electron and phonons is not considered.

Since the energy exchanges are described by heat transfers, the heat diffusion has an important role in the system. In the electronic subsystem, heat diffusion is described by a classical heat diffusion equation. The main parameters of this equation are the electronic specific, $C_{e}$, which is temperature dependent. $C_{e}$ describes the amount of energy needed to increase the electronic temperature of one of the finite element of $1 \mathrm{~K}$. It has no influence on the energy loss of the moving ions, but regulates the amount of energy that will be fed back to the ionic subsystem from the electronic subsystem.

It was observed that the temperature dependent electronic specific heat $C_{e}(x)$ expression implemented within the TTM model in LAMMPS shows a small unrealistic bump [17] before reaching the asymptotic value of $\frac{3}{2} k_{b}$ for high $T_{\mathrm{e}}$ (electronic temperature). Thus, we have implemented our own smoother expression of $C_{e}(x)$ in LAMMPS (with $x=\frac{T_{e}}{1000}$ ):

$$
C_{e}(x)=0.5\left(\frac{3}{2} k_{b}-\epsilon\right)\left[1+\tanh \left(A\left(x-x_{f}\right)\right)\right]+\epsilon
$$

This expression is simpler than the one previously used within LAMMPS [18] as only three free parameters, $\epsilon, A$ and $x_{f}$ have to be determined. Moreover, $\epsilon$ is not a real physical parameter but a computational trick: due to the way the TTM has been implemented within $L A M M P S$, the electronic specific heat must not be equal or very close to zero. The more important TTM parameters employed for Si are displayed in Table I below.

TABLE I

TTM PARAMETERS FOR SI

\begin{tabular}{||c|c|}
\hline \hline$\epsilon$ & $3 \times 10^{-6} \mathrm{eV} \cdot \mathrm{K}^{-1}$ \\
\hline$A$ & 1.3 \\
\hline$x_{f}$ & 3.09 \\
\hline$\gamma_{s}$ & 39.474 g.mol ${ }^{-1} \cdot \mathrm{ps}^{-1}$ \\
\hline$\gamma_{p}$ & 24.443 g.mol ${ }^{-1} \cdot \mathrm{ps}^{-1}$ \\
\hline$v_{0}$ & $79.76 \AA . \mathrm{ps}^{-1}$ \\
\hline
\end{tabular}

For all the materials considered, the parameters $\epsilon, A$ and $x_{f}$ have not been changed. Indeed we found no sufficient experimental data on $\mathrm{Ge}$ and $\mathrm{Si}_{1-\mathrm{x}} \mathrm{Ge}_{\mathrm{x}}$ to justify a change in $C_{e}(x)$. This parameter is not expected to induce major changes in our simulations. The electronic stopping parameters $\gamma_{s}$ were calculated for each system using SRIM [19] tables and the Lindhard and Sharff model [20]. For $\mathrm{Si}_{1-\mathrm{x}} \mathrm{Ge}_{\mathrm{x}}$ systems, the electron-ion coupling parameter $\gamma_{p}$ was kept equal to the value of $\mathrm{Si}$ [21] as we are mainly considering small values of $x$ and we found no trace of electron-phonon coupling parameters for such systems in the literature. The electronic stopping critical velocity $v_{0}$ was taken as being two times the cohesive energy of the system. It is often cited as good practice [22] to turn on electron phonon coupling only after a very short period of system equilibration (usually around $0.2 \mathrm{ps}$ ). This option not being available with LAMMPS yet, the electron-phonon coupling was always turned on.

The calculations were performed for $\mathrm{Si}, \mathrm{Ge}$ and $\mathrm{Si}_{1-\mathrm{x}} \mathrm{Ge}_{\mathrm{x}}$ using the Stillinger-Weber potential, in its original form for $\mathrm{Si}$ [23], with the parameters optimized by K. Nordlund et al. for Ge [24] from the previous work on Ge by K. Ding and C. H. Andersen [25] and using the combination rules of [26] for $\mathrm{Si}_{1}$ ${ }_{x} \mathrm{Ge}_{\mathrm{x}}$. However, in order to describe properly the collisions between atoms, the two-body part of the SW potential has been coupled to the diatomic repulsive Ziegler-BiersackLittmark (ZBL) Universal Potential [27]. The ZBL potential has the following expression:

$$
\begin{gathered}
V_{Z B L}(r)=\frac{Z_{1} Z_{2} e^{2}}{\left(4 \pi \epsilon_{0} r\right)}\left(0.1818 e^{-3.2 \frac{r}{a_{u}}}+0.5099 e^{-0.9432 \frac{r}{a_{u}}}+\right. \\
\left.0.2802 e^{-0.4028 \frac{r}{a_{u}}}+0.2016 e^{-0.02817 \frac{r}{a_{u}}}\right)
\end{gathered}
$$

With:

$$
a_{u}=\frac{0.8854 a_{0}}{Z_{1}^{0.23}+Z_{2}^{0.23}}
$$

Where $Z_{1}$ and $Z_{2}$ are the atomic numbers of atoms 1 and 2. $e$ is the elementary charge, $a_{0}$ the bohr radius and $r$ the interatomic distance between the two atoms considered.

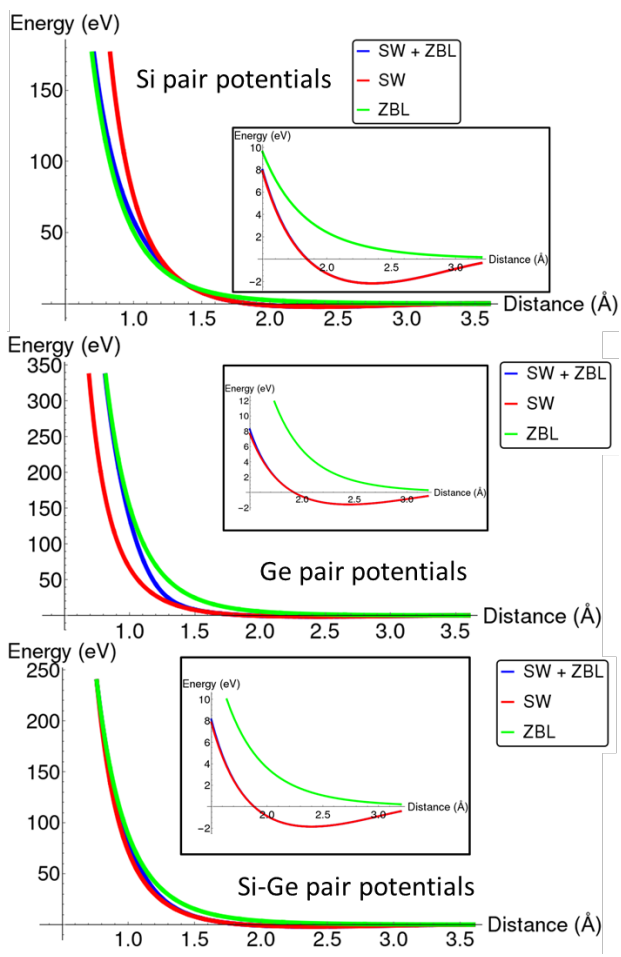

Fig. 2. SW pair potential, ZBL and the combination of the two potentials (SW $+\mathrm{ZBL}$ ) as described above for Si-Si interaction, Ge-Ge and Si-Ge. Zooms on the plots between $1.5 \AA$ and $3.0 \AA$ are inserted. On the zooms, SW and $\mathrm{SW}+\mathrm{ZBL}$ curves are completely equal. 
The two potentials have been combined as suggested by F. Gao. et al. [28]:

$$
\begin{gathered}
V_{t o t}(r)=(1-F(r)) V_{Z B L}(r)+F(r) V_{S W}(r) \\
F(r)=\frac{1}{1+\exp \left(-b_{f}\left(r-r_{f}\right)\right)}
\end{gathered}
$$

Where $b_{f}$ and $r_{f}$ are free parameters of the Fermi function which is used to combine the two potentials, thus they have to be carefully chosen.

Expressing the total two-body potential just like it is done in equation (6) ensures describing the short interatomic interactions with the ZBL repulsive potential while conserving the usual SW potential when atoms are far from each other, as can be seen in Fig. 2. We made sure the transition between ZBL and SW is smooth both for $V_{t o t}(r)$ and its first derivative by choosing adequate $b_{f}$ and $r_{f}$ parameters. We have checked for $\mathrm{Si}, \mathrm{Ge}$ and $\mathrm{Si}_{0.8} \mathrm{Ge}_{0.2}$ the values given by LAMMPS with this modified potential for the lattice parameter, the bulk modulus and the fusion temperature. They are all equal to the usual SW values, which seems consistent with the fact that the ZBL part of the potential is used only when interatomic distances are very short. The parameters $b_{f}$ and $r_{f}$ are $6.8 \AA^{-1}$ and $1.12 \AA$ for Si-Si interaction, $9.5 \AA^{-1}$ and $1.15 \AA$ for Ge-Ge interaction and $10 \AA^{-1}$ and $1.11 \AA$ for Si-Ge interaction.

The behavior of a collision cascade being stochastic, one cannot draw conclusions on a single simulation but needs to end up with statistically meaningful results. So, to ensure good statistics, for each incident energy and each type of PKA, 100 cascades were simulated over 100 non-equivalent directions. For $1 \mathrm{keV}$ cascades, the computational cost being reasonable, we have even set the initial positions of the PKA to both of its inequivalent position (the diamond structure is made of the repetition of a pattern of 2 non-equivalent atoms) and generated multiple random structures for $\mathrm{Si}_{1-\mathrm{x}} \mathrm{Ge}_{\mathrm{x}}$. The number of cascades on which the statistics are calculated and the graphs plotted will be specified.

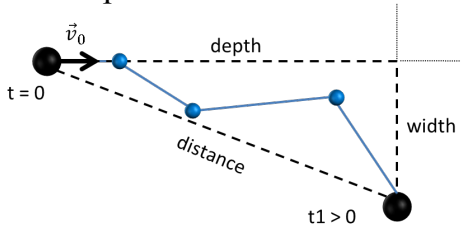

Fig. 3. Vocabulary explanation for the description of a cascade. Black balls represent the PKA, at its initial position and at its position at a time $t_{1}$. Blue balls are the position in space where the PKA shocked another atom.

The main information which can be obtained from the TTM-MD simulations presented above are the penetration depth of the PKAs, the size of the cascade, the number of defects and the aspect of the clusters of defects. Fig. 3 is here to clearly set the vocabulary used to describe a cascade. What is called distance is the length separating the initial position of the PKA with the position of the PKA at the time considered. The depth or penetration depth is the projection of the distance over the direction of the initial velocity of the PKA. The width describes how far the PKA went perpendicularly to the direction of its initial velocity. When values like $<$ distance $>$,
$<$ depth $>$ or $<$ width $>$ will be mentioned it means we are considering the mean value over all the cascades of the maximum distances, depths or widths per cascade. $\operatorname{Var}_{\text {depth }}$ describes the variance of the maximum depth of each cascade regarding the $<$ depth $>$ value.

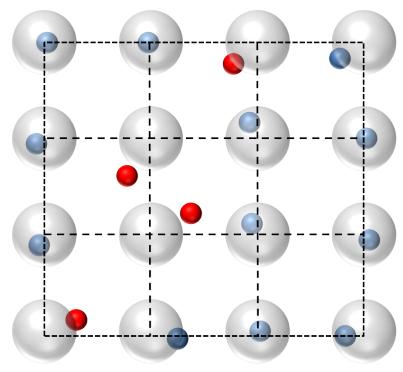

Fig. 4. Representation of the Lindemann criterion for defects. Red spheres are interstitials, blue spheres are atoms near a lattice site and grey spheres are the Lindemann spheres envelope. Black lines cross at lattice sites.

The number of defects is determined following the Lindemann radius criterion (see Fig. 4): if an atom is not located inside any of the Lindemann spheres of radius $r_{L}$ centered on the lattice theoretical sites, it is declared as an interstitial. Consequently, an empty sphere is a vacancy. Therefore, "number of defects" refers to the number of vacancies or interstitials and is not the sum of the two. For Silicon, the radius usually chosen is $0.45 \AA$ [29]. For Ge and $\mathrm{Si}_{1-\mathrm{x}} \mathrm{Ge}_{\mathrm{x}}$ we have simply defined $r_{L}$ proportional to the lattice parameters of the system.

For instance for Germanium:

$$
r_{L}(G e)=a_{\text {lat }}(G e) \times\left(\frac{r_{L}(S i)}{a_{\text {lat }}(S i)}\right)
$$

alat being the lattice parameter of the considered system.

\section{RESUlTS AND DISCUSSIONS}

\section{A. MC: Primary Particle Interaction}

We have investigated the PKA creation of $\mathrm{Si}_{0.8} \mathrm{Ge}_{0.2}$ alloy under neutron irradiation using Geant4. The first striking information given by Fig. 5 showing the frequency of the PKAs created with respect to their energy is that the recoil atoms with the lowest energies are the ones which are the most frequent.

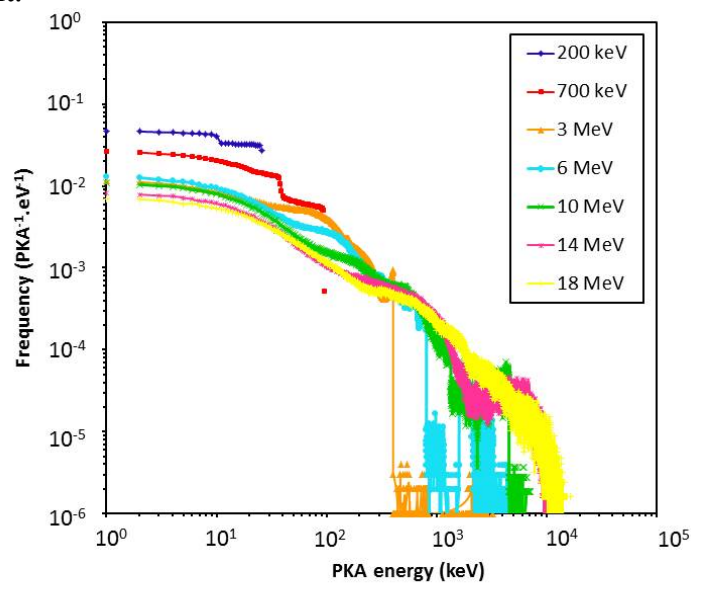

Fig. 5. PKA energy spectra for different energies of incident neutrons in an infinite box of $\mathrm{Si}_{0.8} \mathrm{Ge}_{0.2}$. Nuclear elastic and inelastic interactions considered. 
The lower the incident neutron energies, the flatter the low energy parts of the curves seem to be. Moreover, for $6 \mathrm{MeV}$ neutrons and below we do not observe a smooth decrease of the PKAs frequencies with the increase in PKAs energies but a sudden collapse.

Compared to the previous study carried out on Silicon in [5], [6] and [7] and using the same global simulation approach, where the only type of recoil atoms considered were Silicon atoms, we here have an alloy of Silicon and Germanium. Therefore, for each incident neutron energy, it is worth investigating the types of PKAs created and their relative proportion. As expected, it can be seen in Fig. 6, which shows the percentage of PKA types created depending on the neutron incident energy, that the probability for an atom to undergo a nuclear reaction increases with the energy of the incident neutrons. Nevertheless, even for $18 \mathrm{MeV}$ neutrons, around $20 \%$ of the PKA are $\mathrm{Ge}$ atoms, and $40 \%$ are $\mathrm{Si}$ atoms, meaning that $60 \%$ of all the PKA created are still made of the initial constituents of the alloy. We chose here to focus on $\mathrm{Si}$ and Ge PKA for collision cascade simulations (MD simulations presented later in this article) but do not exclude to focus on other nuclear reactions heavy products in a near future. Indeed, just like it has been stated by Raine et al. [5] light particles are all very high energy particles having very long ranges and depositing very little non-ionizing energy on their path. Therefore we do not expect those light recoils to induce significant DD in the material.

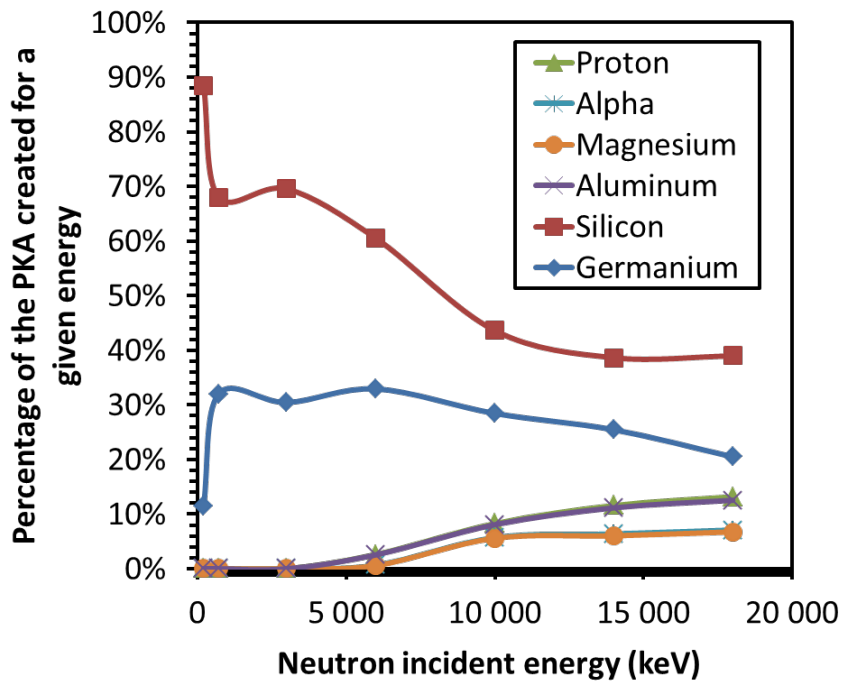

Fig. 6. Evolution in the proportion of the types of PKA atoms created in $\mathrm{Si}_{0.8} \mathrm{Ge}_{0.2}$ for different energies of incident neutrons. Recoils whose proportion did not exceed $1 \%$ are not showed. Nuclear elastic and inelastic interactions considered.

Fig. 6 reveals, except for the $200 \mathrm{keV}$ case (corresponding to a resonance peak for the $\mathrm{Si}-28$ elastic scattering cross section), Ge recoils always contribute between $20 \%$ and $32 \%$ of the PKAs proportion whereas Si recoils range from $42 \%$ to $70 \%$. The investigated material being made of $20 \%$ of Ge, it is not surprising $\mathrm{Ge}$ recoils are less frequent than $\mathrm{Si}$ recoils. Nonetheless, due to spallation reactions and the production of $\mathrm{Mg}, \mathrm{Al}, \alpha$ particles and protons from $\mathrm{Si}$ (at the energies considered we barely found any spallation reaction involving $\mathrm{Ge}$ ), the ratio between $\mathrm{Ge}$ recoils and $\mathrm{Si}$ recoils is always higher than the initial alloy proportion. In Fig. 7 we learn that
Ge PKAs are lower in energy than Si PKAs, the impartation of kinetic energy from the incident neutrons being more effective with lighter atoms. It is also worth noticing that for recoil atoms energies lower than $20 \mathrm{keV}$, it is more frequent to have a Ge PKA than a Si PKA (5 times more frequent for $1 \mathrm{keV}$ recoil atoms originating from $3 \mathrm{MeV}$ neutrons).

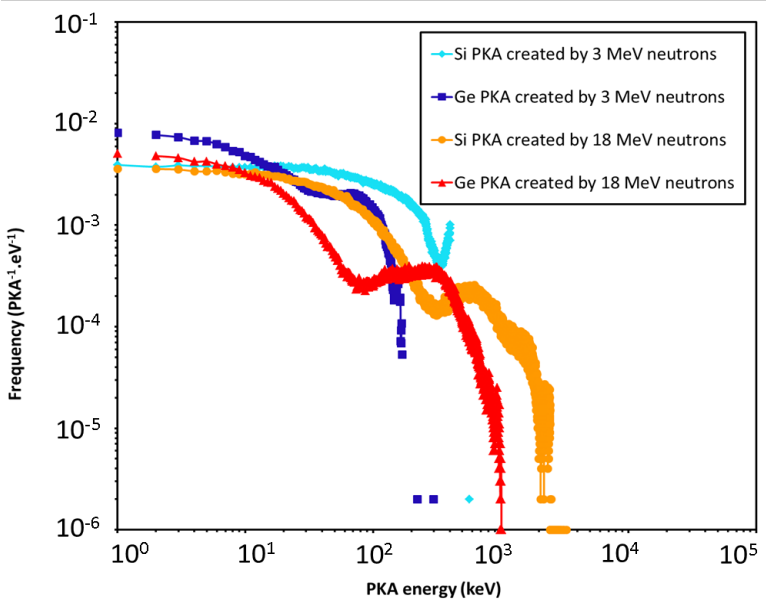

Fig. 7. Comparison between Si PKA and Ge PKA spectra for $3 \mathrm{MeV}$ and 18 $\mathrm{MeV}$ incident neutrons. Nuclear elastic and inelastic interactions considered.

In $\mathrm{Si}$, recoil atoms of $10 \mathrm{keV}$ and less were identified as being representative of the whole collision cascade of defects in [5] as above $10 \mathrm{keV}$ the cascade tends to split into subcascades without overlapping between the subcascades.

$\mathrm{Ge}$ recoil atoms are at least as frequent as $\mathrm{Si}$ recoil atoms when considering energies lower than $20 \mathrm{keV}$ and deposit a large proportion of their energy into non-ionizing event as Fig. 8 attests. They are therefore key contributors to DD and perfectly suitable to be studied with MD simulations.

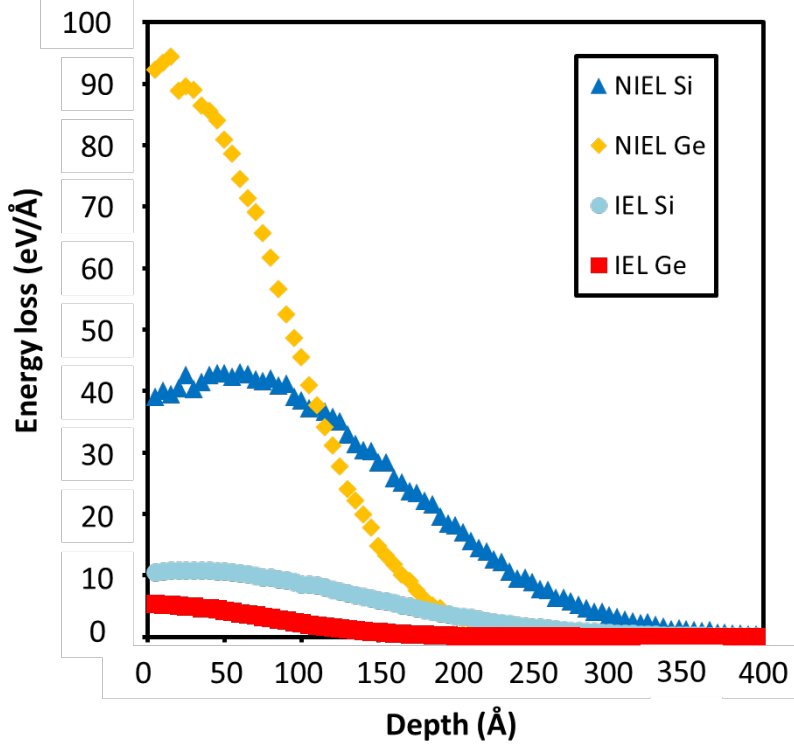

Fig. 8. Non Ionizing Energy Loss (NIEL) and Ionizing Energy Loss (IEL) of $10 \mathrm{keV} \mathrm{Si}$ and Ge PKAs in $\mathrm{Si}_{0.8} \mathrm{Ge}_{0.2}$ versus depth. The calculations have been performed on 10000 cascades for each PKA type with SRIM.

Both Si PKA and Ge PKAs have to be used in the next MD simulation step. Number of defects at the end of the MD cascade as well as penetration depth of the PKAs and range of the cascade are expected to be different depending on the PKA 
type. Indeed, Ge atoms are much heavier and have a different electronic stopping power.

\section{B. MD: Generation of the collision cascades}

A TTM-MD simulated collision cascade is composed of 5 main steps. During the very short first step the PKA shocks atoms and creates high velocity Secondary Knock-On Atoms (SKA). Then those atoms transfer their energy to surrounding atoms, creating slow moving atoms. The third step is the creation of Frenkel pairs in the structure, followed by the propagation of an elastic thermal wave. Finally, a non negligible part of the Frenkel pairs will recombine.

Here we focused on the comparison between collision cascades performed on $\mathrm{Si}, \mathrm{Ge}$ and on $\mathrm{Si}_{1-\mathrm{x}} \mathrm{Ge}_{\mathrm{x}}$ alloys, aiming at highlighting the different DD response of those materials under irradiation or ion implantation. We expect to find at the end of the simulation more damage in $\mathrm{Si}_{1-\mathrm{x}} \mathrm{Ge}_{\mathrm{x}}$ alloys than in $\mathrm{Si}$, but less than in Ge. It is worth mentioning that the amount of damage found at the end of the TTM-MD cascade is not representative of the damage measured during experiments. The damage structures would have to be relaxed with k-ART before being able to compare them with experimental data. Nonetheless, even if the damage is overestimated with TTM$\mathrm{MD}$, it is already a very good clue on the DD mechanisms at stake in $\mathrm{Si}_{1-\mathrm{x}} \mathrm{Ge}_{\mathrm{x}}$.

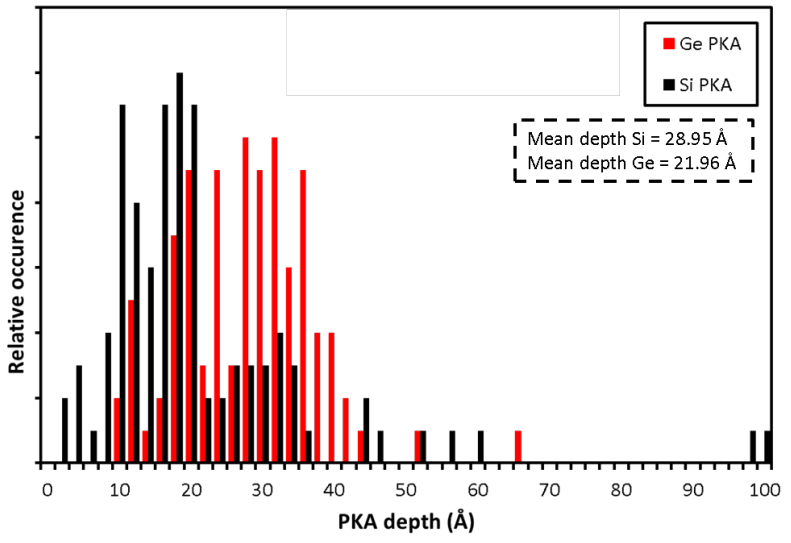

Fig. 9. Penetration depth of $1 \mathrm{keV}$ Si and Ge PKA in $\mathrm{Si}_{0.8} \mathrm{Ge}_{0.2}$. Simulations were performed with SW potential only.

Just for the sake of comparison we present here results that were obtained using SW only as an interatomic potential (instead of our potential combining SW and ZBL). We observed in Fig. 9, which shows the relative occurrence of the depth of the PKAs, using the SW potential only that Ge PKAs were found deeper in $\mathrm{Si}_{1-\mathrm{x}} \mathrm{Ge}_{\mathrm{x}}$ alloys than $\mathrm{Si}$ PKAs. On top of that, it was found that self recoil atoms in pure Ge penetrated deeper in the material than Si self recoils, for the same energy. However, we expected the inverse trend and SRIM as well as experimental studies [30] tend to show that the lighter atoms travel further in the material, therefore in disagreement with what we have obtained.

Many tests have been carried out trying to find the part of the model which was responsible of those surprising results. We started by questioning the choice of the TTM parameters and the SW potential parameters. We found using SRIM 2008 a higher electronic stopping power for $\mathrm{Si}$ in $\mathrm{Si}_{0.8} \mathrm{Ge}_{0.2}$ than for $\mathrm{Ge}$ in $\mathrm{Si}_{0.8} \mathrm{Ge}_{0.2}$. Even if at $1 \mathrm{keV}$ the TTM should not induce major differences (the stopping force is proportional to the speed of the recoil atoms), we thought it would be worth investigating the influence of the TTM on the cascade development. However, it quickly turned out that despite how significant the choice of those parameters is for the simulations, the explanation of our observations could not lie upon it.

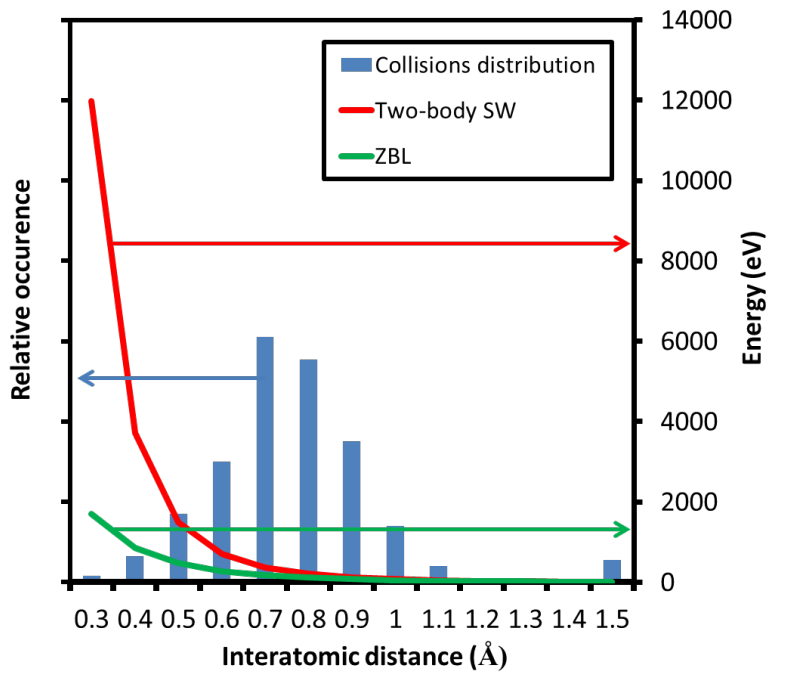

Fig. 10. Distribution of the collisions involving the PKA over 100 simulations performed on Si with $1 \mathrm{keV}$ Si PKA. The SW and ZBL potentials are superimposed. Arrows are showing to which $\mathrm{Y}$ axis the curves and histogram refer to.

The potential itself was the only parameter we had not tested yet and which could explain the odd results obtained. Moreover, some studies already highlighted the significance of the repulsive potential when carrying out collision cascades simulations [31], [32]. SW potential has already proven itself to be very precise and efficient concerning equilibrium properties. However, for high energies particles during the collision phase, the interatomic distances can become very short. The transferability of the SW potential in such extreme cases is thus questionable. To explain the unrealistic results obtained with the SW potential we have examined the typical interatomic distances between the PKA and another atom in case of shock in order to see if distances too short for the SW potential to be perfectly suited to our cases of interest are attained. Those simulations (usual set of 100 simulations) were performed on Si with Si PKA of $1 \mathrm{keV}$. Results are displayed in Fig. 10. According to our findings, even at $1 \mathrm{keV}$ only, very short interatomic distances are reached. For 100 simulations, 465 shocks involving the PKA and an interatomic distance of less than $1.5 \AA$ have been counted. Among those shocks, a large majority involved interatomic distances of $1 \AA$ or lower. As can be seen in Fig. 10, the large majority of the collisions occurred in a region where the discrepancy between the $\mathrm{SW}$ and ZBL potentials is significant.

Following this, a large amount of $1 \mathrm{keV}$ simulations were performed on $\mathrm{Si}, \mathrm{Si}_{0.8} \mathrm{Ge}_{0.2}, \mathrm{Si}_{0.7} \mathrm{Ge}_{0.3}, \mathrm{Si}_{0.5} \mathrm{Ge}_{0.5}$ and $\mathrm{Ge}$ using our modified potential. The differences with the simulations carried out using the SW potential only are obvious. Focusing exclusively on $1 \mathrm{keV} \mathrm{Si}$ PKA in Si and $1 \mathrm{keV}$ Ge PKA in Ge (Fig. 11), the results obtained with the modified SW potential give a penetration depth in Si by far superior to the one in $\mathrm{Ge}$, which is much more in agreement with SRIM results. 


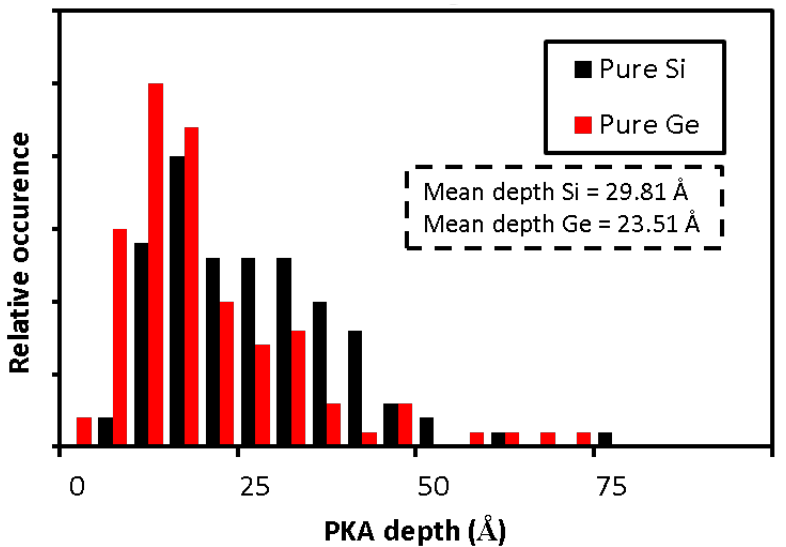

Fig. 11. Penetration depth of Si and Ge $1 \mathrm{keV}$ PKA in respectively pure Si and pure Ge. 100 simulations per atom types were performed.

Calculation results obtained with $1 \mathrm{keV}$ PKAs and using SW combined with ZBL are summed up in Table II. In $\mathrm{Si}_{1}$ ${ }_{x} \mathrm{Ge}_{\mathrm{x}}$ alloys, the differences in the penetration depth of $\mathrm{Si}$ PKAs compared with Ge PKAs are a lot smaller than in pure $\mathrm{Si}$ and pure Ge. One could argue that such small differences might only be simulation artifacts but those tiny differences were always observed for every set of 200 simulations performed (100 simulations with Si PKAs for 100 nonequivalent directions, same for Ge PKA). Moreover, SRIM also gives small differences in the penetration depth for relatively low recoil energies like $1 \mathrm{keV}$. To us, the comparison with another code and the large statistic ensembles used are enough to assess the confidence in the trustfulness of our results.

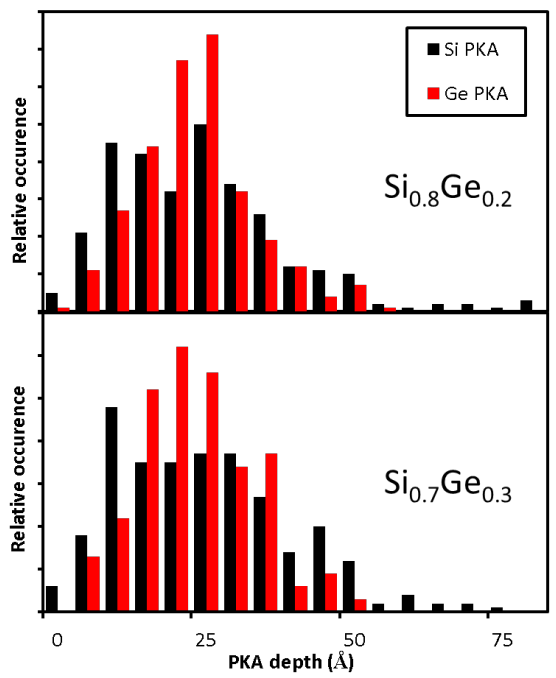

Fig. 12. Penetration depth of $\mathrm{Si}$ and $\mathrm{Ge} 1 \mathrm{keV}$ PKA in $\mathrm{Si}_{0.8} \mathrm{Ge}_{0.2}$ and $\mathrm{Si} 0.7 \mathrm{Ge} 0.3 .300$ simulations per PKA types were performed.

Regarding the distribution of PKAs penetration depth, it can be seen on Fig. 12 that the values for Si PKAs are much more spread than the ones for Ge PKAs. Si PKAs penetration depths data gathering the lowest and highest value whereas Ge PKAs penetration depth seem more centered on a mean value. This is confirmed by the row in Table. II showing the variance of the PKA penetration depth. Another interesting observation is the width of the PKAs depending on the PKAs type. The mean width of the PKAs trajectories is a lot larger in the case of $\mathrm{Si}$ PKA than in the case of Ge PKAs.

TABLE II

Calculated Mean Values of TyPical Cascade Parameters Over 100 SIMULATIONS PER PKA TYPE FOR PURE MATERIALS AND 300 SiMULATIONS PER PKA TYPE AND Alloy COMPOSITION FOR AlLOYS.

\begin{tabular}{|c|c|c|c|c|}
\hline & $<$ distance $>(\AA)$ & $<\operatorname{depth}>(\AA)$ & $<$ width $>(\AA)$ & Var $_{\text {depth }}$ \\
\hline $\begin{array}{l}\text { Pure Si } \\
\text { Si PKA }\end{array}$ & 29.81 & 27.18 & 11.75 & 12.63 \\
\hline $\begin{array}{l}\text { Pure Ge } \\
\text { Ge PKA }\end{array}$ & 23.51 & 20.49 & 11.12 & 13.29 \\
\hline $\begin{array}{c}\mathrm{Si}_{0.8} \mathrm{Ge}_{0.2} \\
\text { Si PKA }\end{array}$ & 30.58 & 26.59 & 14.14 & 14.57 \\
\hline $\begin{array}{l}\mathrm{Si}_{0.8} \mathrm{Ge}_{0.2} \\
\text { Ge PKA }\end{array}$ & 28.02 & 25.53 & 10.59 & 9.52 \\
\hline $\begin{array}{c}\mathrm{Si}_{0.7} \mathrm{Ge}_{0.3} \\
\text { Si PKA }\end{array}$ & 31.77 & 27.50 & 15.04 & 14.72 \\
\hline $\begin{array}{l}\mathrm{Si}_{0.7} \mathrm{Ge}_{0.3} \\
\text { Ge PKA }\end{array}$ & 29.46 & 26.46 & 12.19 & 11.71 \\
\hline $\begin{array}{c}\mathrm{Si}_{0.5} \mathrm{Ge}_{0.5} \\
\text { Si PKA }\end{array}$ & 30.96 & 26.23 & 15.85 & 14.73 \\
\hline $\begin{array}{l}\mathrm{Si}_{0.5} \mathrm{Ge}_{0.5} \\
\text { Ge PKA }\end{array}$ & 28.05 & 25.30 & 11.53 & 11.53 \\
\hline
\end{tabular}

According to the observations of the trajectory of various cascades it seems that $\mathrm{Si}$ PKAs, due to their low mass compared to Ge atoms, tend to deviate significantly from their trajectory when they collide with a Ge atom. On the contrary, Ge PKAs trajectories are not significantly disturbed by $\mathrm{Si}$ atoms. This is what could explain the enhance width of $\mathrm{Si}$ PKAs trajectories in Si-Ge alloys as well as the significant spreading of Si PKAs depths.

The amount of damage is expected to increase with increasing fraction of Ge in the alloy. It is indeed observed that the lowest number of defects after the end of the $100 \mathrm{ps}$ TTM-MD cascade is found in pure $\mathrm{Si}$, the highest in pure $\mathrm{Ge}$ and that the number of defects increases going from pure Si to pure Ge (both for Si and Ge PKAs). It is also found that Ge PKAs creates more defects than Si PKAs (see table IV). This result concerning the amount of damage remaining at the end of the cascade follows the expected trend but it is hard to be totally conclusive with $1 \mathrm{keV}$ simulations only.

TABLE III

CALCULATED MEAN VALUES OF TYPICAL CASCADE PARAMETERS OVER 100 SIMULATIONS PER PKA TYPE AND ENERGY.

\begin{tabular}{|c|c|c|c|c|}
\hline & $<$ distance $>(\AA)$ & $<$ depth $>(\AA)$ & $<$ width $>(\AA)$ & Vardepph \\
\hline $\begin{array}{c}\text { Pure } \mathrm{Si} \\
5 \mathrm{keV}\end{array}$ & 102.5 & 93.2 & 40.3 & 57.9 \\
\hline $\begin{array}{c}\text { Pure Ge } \\
5 \mathrm{keV}\end{array}$ & 56.7 & 49.1 & 27.4 & 27.3 \\
\hline $\begin{array}{c}\mathrm{Si} .0 .8 \mathrm{Ge} .2 \mathrm{Si} \text { PKA } \\
5 \mathrm{keV}\end{array}$ & 96.6 & 84.0 & 43.8 & 51.6 \\
\hline $\begin{array}{c}\mathrm{Si}_{0.8} \mathrm{Ge}_{0.2} \\
\mathrm{Ge} \mathrm{PKA} \\
5 \mathrm{keV} \\
\end{array}$ & 67.0 & 60.9 & 25.3 & 26.5 \\
\hline $\begin{array}{l}\mathrm{Si}_{0.8 \mathrm{Ge}} \mathrm{Ge} \\
\mathrm{Si} \mathrm{PKA} \\
10 \mathrm{keV}\end{array}$ & 133.2 & 118.0 & 60.6 & 59.6 \\
\hline $\begin{array}{l}\mathrm{Si} .0 \mathrm{ge}_{0.2} \\
\mathrm{Ge} \mathrm{PKA} \\
10 \mathrm{keV}\end{array}$ & 99.8 & 91.8 & 35.5 & 41.9 \\
\hline
\end{tabular}


Concerning $5 \mathrm{keV}$ and $10 \mathrm{keV}$ cascade simulations, the trends forecasted thanks to $1 \mathrm{keV}$ calculations is confirmed and even emphasized. The discrepancies in the cascade development in $\mathrm{Si}_{0.8} \mathrm{Ge}_{0.2}$ depending on the type of the PKAs are now blatant. The penetration depth is obviously highly superior for Si PKAs than Ge PKAs, as well as the width of the trajectories starting with a Si PKAs and the spreading of $\mathrm{Si}$ PKAs penetration depth. Data summing up all those cascades parameters for 5 and $10 \mathrm{keV}$ cascades are displayed in Table III. The damage analysis performed on $5 \mathrm{keV}$ and $10 \mathrm{keV}$ collision cascades final structures (after 2 ns of TTM-MD) whose results are available in Table IV confirmed our hypothesis formulated after the $1 \mathrm{keV}$ simulations i.e. structures are much more damaged after Ge PKA cascades and the higher the Ge fraction the higher the level of damage. The methods for counting defects from MD simulations being very diverse in nature very different results can be obtained. Therefore, the results provided in Table IV should not be considered as definitive results on the defects that can be found in $\mathrm{Si}, \mathrm{Ge}$ and $\mathrm{Si}-\mathrm{Ge}$ alloys but as the results obtained with the specific counting technique we have chosen. However, the trends in the evolution of the number of defects are consistent between all the methods available. A detailed benchmarking of the discrepancies between techniques has been performed by K. Nordlund et al. in [24].

TABLE IV

MEAN NuMBER OF DEFECTS AT THE END OF THE CASCADES FOR VARIOUS ENERgies AND Systems (100 Simulations Per PKa TyPe AND ENERGy).

\begin{tabular}{|c||c|c|c|c||}
\hline & Pure Si & Pure Ge & $\begin{array}{l}\mathrm{Si}_{0.8} \mathrm{Ge}_{0.2} \\
\text { Si PKA }\end{array}$ & $\begin{array}{l}\mathrm{Si}_{0.8} \mathrm{Ge}_{0.2} \\
\text { Ge PKA }\end{array}$ \\
\hline \hline $1 \mathrm{keV}$ & 60 & 128 & 82 & 109 \\
\hline $5 \mathrm{keV}$ & 292 & 999 & 395 & 657 \\
\hline $10 \mathrm{keV}$ & $\mathrm{X}$ & $\mathrm{X}$ & 733 & 1274 \\
\hline
\end{tabular}

It is of common knowledge that ion implantation and high energy particles codes like SRIM and Geant 4 are not adapted to low energy calculations like $1 \mathrm{keV}$ PKAs. However, they start to be trustworthy when considering higher energies like 5 $\mathrm{keV}$ and $10 \mathrm{keV}$. Therefore, it makes sense to compare our TTM-MD (modified SW) results with those two MC codes. We have performed some simulations with Geant4 starting with $5 \mathrm{keV}$ and $10 \mathrm{keV} \mathrm{Si}$ and Ge PKAs in $\mathrm{Si}_{0.8} \mathrm{Ge}_{0.2}$ as well as in pure $\mathrm{Si}$ and pure Ge. Fig. 13 provides a comparison between LAMMPS and Geant4, showing the relative occurrence of PKAs depth for the two simulation methods. It can be seen that the distribution of Ge PKAs within $\mathrm{Si}_{0.8} \mathrm{Ge}_{0.2}$ is pretty similar between the 2 methods: very few PKAs penetrating no deeper than a few tens of Angstroms, a large majority of PKA between $50 \AA$ and $150 \AA$ and almost no PKAs going deeper than $250 \AA$. This apparent similarity is confirmed in Table $\mathrm{V}$ by the calculation of cascade mean typical values.
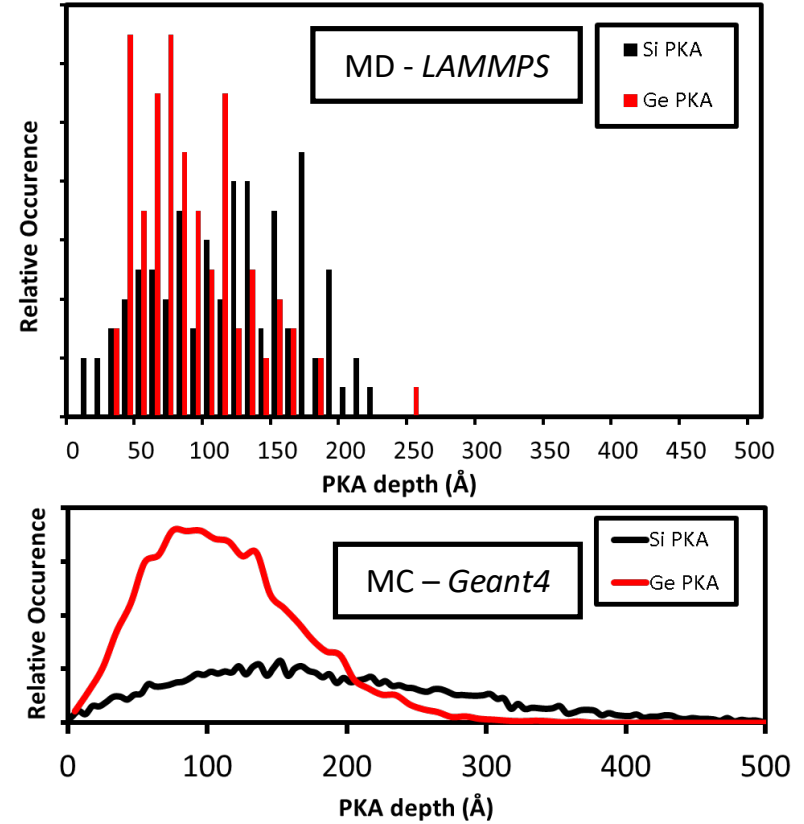

Fig. 13. PKA penetration depth distribution in $\mathrm{Si}_{0.8} \mathrm{Ge}_{0.2}$ starting from $10 \mathrm{keV}$ Si and Ge PKA obtained with LAMMPS and Geant4.

Concerning Si PKAs, Fig. 13 shows a distribution much broader with Geant4 than with LAMMPS. We had already noticed a very broad spectra of Si PKAs penetration depths but never as large as the one of Fig. 13. MC part. A non negligible number of PKAs penetrate even deeper than $250 \AA$ in the material, which is the maximum observed with LAMMPS. If there is no doubt the two distributions are quite different, the reason we do not find PKAs deeper than $250 \AA$ with LAMMPS is that we are limited by the size of our box: we could expect a PKA to travel at maximum between $250 \AA$ and $300 \AA$ A before leaving the box.

TABLE V

MEAN PKA DEPTH CALCULATED WITH LAMMPS, GEANT4 AND SRIM For VARIOUS ENERGIES AND TARgET MATERIALS. LAMMPS VALUES ARE AVERAGED OVER 100 SiMULATIONS PER TARGET MATERIAL AND ENERGY, GEANT4 OVER 10000 AND SRIM OVER 5000.

\begin{tabular}{|c|c|c|c|c|c|c|}
\hline & \multicolumn{3}{|c|}{$\begin{array}{c}\text { Depth }(\AA) \\
5 \mathrm{keV}\end{array}$} & \multicolumn{3}{|c|}{$\begin{array}{c}\text { Depth }(\AA) \\
10 \mathrm{keV}\end{array}$} \\
\hline & $L A M M P S$ & SRIM & Geant4 & LAMMPS & SRIM & Geant4 \\
\hline Pure Si & 93 & 110 & 107 & $\mathrm{X}$ & 192 & 180 \\
\hline Pure Ge & 49 & 54 & 54 & $\mathrm{X}$ & 88 & 84 \\
\hline $\mathrm{Si}_{0.8} \mathrm{Ge}_{0.2} \mathrm{Si}$ PKA & 84 & 107 & 102 & 118 & 185 & 172 \\
\hline $\mathrm{Si}_{0.8} \mathrm{Ge}_{0.2} \mathrm{Ge}$ PKA & 61 & 67 & 76 & 92 & 114 & 117 \\
\hline
\end{tabular}


For $10 \mathrm{keV}$ Si PKAs in $\mathrm{Si}_{0.8} \mathrm{Ge}_{0.2}$ a few cascades went out of the box of 1000000 atoms and had to be simulated again starting with another initial PKA position in order to make the PKAs and the extensions of the cascades stay inside the box. This means that for a few simulations, the PKAs could have had a depth larger than $250 \AA$ but the size of our TTM-MD box compelled us to choose not to include those PKA in our statistics. Choosing a box whose size would be large enough so that none of the cascades will never go out of the box would be too time and computationally consuming. This is important to keep in mind but only concerns a very small number of cascades of the $\mathrm{Si}_{0.8} \mathrm{G}_{\mathrm{e} 0.2} 10 \mathrm{keV}$ Si PKAs set of cascades only.

TABLE VI

DEFECTS Distribution DEPENDING ON THE CLUSTER SizES FOR VARIOUS ENERGIES, MATERIALS AND PKA TYPES.

\begin{tabular}{||c||c||c|c||c||}
\hline \hline & $\begin{array}{c}\text { Mean } \\
\text { number of } \\
\text { clusters per } \\
\text { cascade }\end{array}$ & $\begin{array}{c}\% \text { of } \\
\text { defects in } \\
\text { small } \\
\text { clusters }\end{array}$ & $\begin{array}{c}\% \text { of defects } \\
\text { in medium } \\
\text { clusters }\end{array}$ & $\begin{array}{c}\% \text { of defects } \\
\text { in large } \\
\text { clusters }\end{array}$ \\
\hline \hline $\begin{array}{c}5 \mathrm{keV} \\
\text { Pure Si }\end{array}$ & 18 & $12 \%$ & $59 \%$ & $29 \%$ \\
\hline $\begin{array}{c}5 \mathrm{keV} \\
\text { Pure Ge }\end{array}$ & 13 & $3 \%$ & $8 \%$ & $89 \%$ \\
\hline $\begin{array}{c}5 \mathrm{keV} \\
\mathrm{Si} \mathrm{PKA}\end{array}$ & 18 & $11 \%$ & $39 \%$ & $50 \%$ \\
\hline $\begin{array}{c}5 \mathrm{keV} \\
\mathrm{Ge} \text { PKA }\end{array}$ & 12 & $4 \%$ & $13 \%$ & $83 \%$ \\
\hline $\begin{array}{c}10 \mathrm{keV} \\
\mathrm{Si} \mathrm{PKA}\end{array}$ & 32 & $10 \%$ & $36 \%$ & $54 \%$ \\
\hline $\begin{array}{c}10 \mathrm{keV} \\
\mathrm{Ge} \mathrm{PKA}\end{array}$ & 22 & $4 \%$ & $11 \%$ & $85 \%$ \\
\hline \hline
\end{tabular}

Table $\mathrm{V}$ shows the simulations performed with $L A M M P S$ always underestimate the PKA depth compared to Geant4 and SRIM. However, the agreement between TTM-MD simulated values and MC calculations are very satisfactory, except for the $10 \mathrm{keV}$ Si PKAs in $\mathrm{Si}_{0.8} \mathrm{Ge}_{0.2}$ value. In this particular case, there is a clear discrepancy between LAMMPS on one side and Geant4 and SRIM on the other side which we already discussed.

Carrying out higher energy simulations not only confirmed the trends observed for lower energies but allowed the study of more complicated phenomena which could not be observed at low energy and involving the need for an atomistic description of the matter and many-body interaction potentials, therefore not attainable with $\mathrm{MC}$ techniques. Among those phenomena, an important one is the formation of clusters of defects. Clusters are defined as isolated defects and aggregates of defects (interstitials and vacancies) which are separated from another defect of the cluster by a at most 2 times the shortest distance between atoms in the lattice (i.e. $2 \times a \sqrt{3} / 4, a$ being the lattice parameter). We define small clusters as containing between 0 (vacancy) to 5 interstitials, medium clusters as clusters containing from 5 to 100 interstitials and large clusters as being made of more than 100 interstitials atoms.

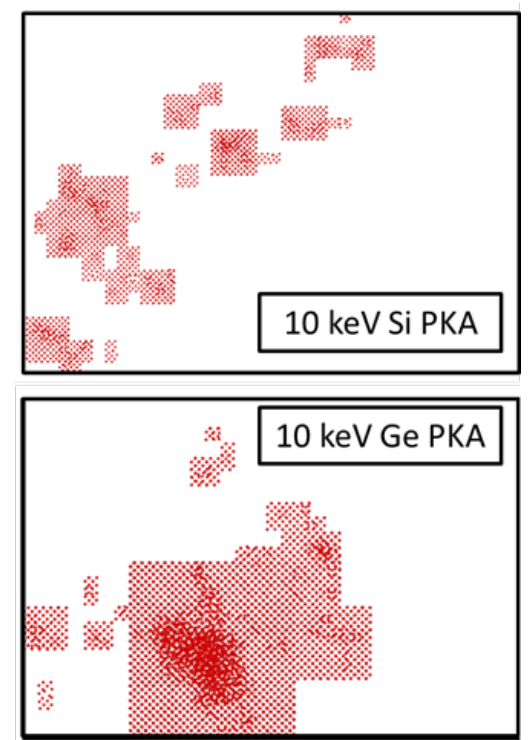

Fig. 14. Final defects observed in $\mathrm{Si}_{0.8} \mathrm{Ge}_{0.2}$ after $10 \mathrm{keV}$ Si PKA simulations (above) and $10 \mathrm{keV} \mathrm{Ge} \mathrm{PKA} \mathrm{(below).} \mathrm{Ge} \mathrm{and} \mathrm{Si}$ atoms have the same color to make the defects and clusters easier to compare. There are around 400 defects in the Si PKA cascade and 1200 in the Ge cascade.

It is much more frequent to find large clusters with cascades starting with Ge PKAs: cascades initiated with a Si PKA will split up, forming isolated defects as well as medium clusters, large clusters and amorphized regions whereas with Ge PKA many cascades are made of one big cluster and very few isolated defects and small clusters around it. This observation is illustrated by Fig. 14 displaying the clusters obtained after $10 \mathrm{keV}$ Si and Ge PKAs simulations in $\mathrm{Si}_{1-\mathrm{x}} \mathrm{Ge}_{\mathrm{x}}$.

Ge PKAs creates shorter and denser cascades as attests their low depth and width and their high number of defects. The energy of Ge PKAs being transmitted over smaller volumes than Si PKAs, the defects are for most of them gathered in large first melted liquid-like and then amorphous zones forming large clusters. The reasons for the discrepancies in the cascade behaviors between pure $\mathrm{Si}$ and pure Ge have been attributed to the reduce defects mobility in Ge, a lower melting point and a lower thermal conductivity [33]. It seems satisfactory reasons as well to explain the increase in the number of defects with the increasing Ge fraction in the Si-Ge. However it cannot explain the two cascades behaviors observed depending on the PKA type for Si and Ge. The two scenarios observed with $\mathrm{Si}$ and Ge PKAs have to deal with the way $\mathrm{Si}$ and $\mathrm{Ge}$ deposit energy on their path i.e. the fact $\mathrm{Ge}$ deposit more energies through shocks and in faster way than $\mathrm{Si}$. It is also interesting to see the discrepancy in clusters distribution between $5 \mathrm{keV}$ Si PKAs in pure $\mathrm{Si}$ and $5 \mathrm{keV} \mathrm{Si}$ PKAs in $\mathrm{Si}_{0.8} \mathrm{Ge}_{0.2}$. It is a lot more probable for a defect to be in a large amorphous cluster in $\mathrm{Si}_{0.8} \mathrm{Ge}_{0.2}$ than it is in pure $\mathrm{Si}$. It was also noticed that large clusters are deficient in interstitial atoms for $80 \%$ and $88 \%$ of them for $\mathrm{Si}$ and Ge $5 \mathrm{keV}$ PKAs, respectively, in $\mathrm{Si}_{0.8} \mathrm{Ge}_{0.2}$. As a matter of fact, small clusters display in most cases (around 70\% for both Si and Ge PKAs) an excess of interstitial atoms. 


\section{Discussion on the comparison between MC, TTM-MD and experiments}

At this stage of the simulation approach defined in Fig. 1, comparison with experimental results is nearly impossible to our knowledge. Indeed, no experimental data describing the complex dynamic mechanisms happening inside the matter on such short timescales is available in literature. Nonetheless, if definite values cannot be compared with experiments, the trends we are observing concerning the number of defects with the fraction of Ge in the alloy, which have been confirmed by experiments, is a first experimental validation [1], [2], [3]. So far, the only value-to-value comparison point we have is with MC codes such as SRIM and Geant4. Those codes have been extensively compared and benchmarked with experimental results and are often considered as reference points even in experimental papers. The good agreement between MD and $\mathrm{MC}$ is therefore a key factor to assess the validity of our results. Therefore, we believe the good comparison between our results and trusted MC codes, as well as trends in the evolution of the number of defects with the fraction of $\mathrm{Ge}$ in the alloy being similar to experimental observations and the benchmarking of the ZBL and SW potentials by the community for diverse applications are enough arguments to guarantee a satisfactory description of the mechanisms observed with TTM-MD.

\section{CONCLUSION}

To describe the displacement damage in $\mathrm{Si}_{1-\mathrm{x}} \mathrm{Ge}_{\mathrm{x}}$, we use an original approach coupling Monte Carlo and Molecular Dynamics coupled to the Two-Temperature Model. This approach allows taking into account the electronic stopping power in MD simulations. The preliminary MC study helped us targeting specific PKA energies and types to be used as input values for the TTM-MD cascade simulations. A large library of damaged structures has been constructed. The results of the analysis of those structures were compared to experimental observations and other simulation codes results. It appeared we managed to computationally forecast some trends experimentally observed concerning the amount of damage in Si-Ge alloys and found values in agreement with SRIM and Geant4 codes, giving us confidence in our results. Nonetheless, it would be interesting to construct a more precise potential for repulsive interactions than the ZBL potential which can lack of accuracy [34]. The remaining structures at the end of MD simulations would have to be annealed and the electronic structures of the most abundant defects investigated with ab initio methods.

\section{ACKNOLEDGMENTS}

The authors would like to thank M. Gaillardin, P. Paillet and L. Martin-Samos for fruitful discussions.

\section{REFERENCES}

[1] T. E. Haynes, and O. W. Holland, "Damage accumulation during ion implantation of unstrained $\mathrm{Si}_{1-\mathrm{x}} \mathrm{Ge}_{\mathrm{x}}$ alloy layers," Appl. Phys. Lett., vol. 61, no. 1, pp. 61-63, July 1992 .

[2] C. O'Raifeartaigh et al., "2 MeV Si ion implantation damage in relaxed $\mathrm{Si}_{1-}$ хGe $\mathrm{x}_{\mathrm{x}}$ " Nucl. Instr. and Methods Phys. Res. B, vol. 120, no. 1-4, pp. 165-168, Dec. 1996.

[3] A. N. Larsen et al., "MeV ion implantation induced damage in relaxed $\mathrm{Si}_{1-}$ xGe ," J. App. Phys., vol. 81, no. 5, pp. 2208-2218, Apr. 1997.

[4] D.Y.C. Lie, "Doping and processing epitaxial $\mathrm{Ge}_{x} \mathrm{Si}_{1-\mathrm{x}}$ Films on $\mathrm{Si}(100)$ by ion implantation for Si-based heterojunction devices applications," Journal of Electronic Materials, vol. 27, no. 5, pp. 377-401, May 1998.

[5] M. Raine et al., "Simulation of single particle displacement damage in silicon - Part I: Global approach and primary interaction simulation," IEEE Trans. Nucl. Sci., vol. 64, no. 1, pp. 133-140, Jan. 2017.

[6] A. Jay et al., "Simulation of single particle displacement damage in silicon - Part II: Generation and Long-Time relaxation of damage structure," IEEE Trans. Nucl. Sci., vol. 64, no. 1, pp. 141-148, Jan. 2017.

[7] A. Jay et al., "Simulation of single particle displacement damage in silicon - Part III: First principle characterization of defect properties," IEEE Trans. Nucl. Sci., vol. 65, no. 2, pp. 724-731, Feb. 2018.

[8] D. Duffy and A. Rutherford, "Including the effect of electronic stopping and electronic-ion interactions in radiation damage simulations," J. Phys,: Condens. Matter, vol. 19, no. 1, p. 016207, Dec. 2006.

[9] L. K. Béland, et al. "Kinetic-activation relaxation technique," Phys. Rev. E, vol. 84 , no. 4 , p. 046704 , Oct. 2011.

[10] R. Malek and N. Mousseau, "Dynamics of Lennard-Jones clusters: A characterization of the activation-relaxation technique," Phys. Rev. E, vol. 62, no. 6, pp. 7723-7728, Jan. 2001.

[11] S. Agostinelli et al., "Geant4 - a simulation toolkit," Nucl. Instrum. and Methods Phys. Res. A, vol. 506, no. 3, pp. 250-303, July 2003.

[12] J. Allison, et al., "Geant4 developments and applications," IEEE Trans. Nucl. Sci., vol. 53, no. 1, pp. 270-278, Feb. 2006.

[13] J. Allison, et al., "Recent Developments in Geant4," Nucl. Instrum. and Methods Phys. Res. A, vol. 853, pp. 186-225, Nov. 2016.

[14] S. Plimton, "Fast parallel algorithms for short-range molecular dynamics," J. Comput. Phys., vol. 117, no. 1, pp. 1-19, Mar. 1995. Available on http://lammps.sandia.gov.

[15] A. Caro and M. Victoria, "Ion-electron interaction in molecular-dynamics cascades," Phys. Rev. A, vol. 40, no. 5, pp. 2287-2291, Sept. 1989.

[16] I. Koponen, "Energy transfer between electrons and ions in dense displacement cascades," Phys. rev. B, vol. 47, no. 21, pp. 14011-14019, June 1993.

[17] A. Akkerman, M. Murat and J. Barak, "Insight into the dynamics of electrons ejected by energetic ions in silicon and its relation to the basics of the inelastic thermal spike model," Nucl. Instrum. and Methods Phys. Res. B, vol. 432, pp. 29-36, Oct. 2018.

[18] LAMMPS TTM webpage available at: https://lammps.sandia.gov/doc/fix_ttm.html

[19] J. F. Ziegler, M. D. Ziegler and J. B. Biersack, "SRIM - The stopping range of ions in matter," Nucl. Instrum. and Methods Phys. Res. B, vol. 268, no. 11-12, pp. 1818-1823, June 2010.

[20] J. Lindhard and M. Sharff, "Energy loss in matter by fast particles of low charge," Mathematisk-Fysiske Meddelelser det Kongelige Danske Videnskabernes Selskab, vol. 27, no. 15, pp. 1-31, 1953.

[21] M. Toulemonde, C. Dufour, A. Meftah and E. Paumier, "Transient thermal processes in heavy ion irradiation of crystalline inorganic insulators," Nucl. Instrum. and Methods Phys. Res. B, vols. 166-167, pp. 903-912, May 2000.

[22] E. Zarkadoula, G. Samolyuk and W. J. Weber, "Effects of the electronphonon coupling activation in collision cascades," J. Nucl. Mater., vol. 490, pp. 317-322, July 2017.

[23] F. H. Stillinger and T. A. Weber, "Computer Simulation of local order in condensed phases of silicon," Phys. Rev. B, vol. 31, no. 8, pp. 5262-5271, Apr. 1985.

[24] K. Nordlund et al., "Defect production in collision cascades in elemental semiconductors and fcc metals," Phys. Rev. B, vol. 57 no. 13, pp. 7556-7570, Apr. 1998.

[25] K. Ding and H. C. Andersen, "Molecular-dynamics simulation of amorphous germanium," Phys. Rev. B, vol. 34, no. 10, pp. 6987-6991, Nov. 1986. 
[26] M. Laradji, D. P. Landau and B. Dünweg, "Structural properties of $\mathrm{Si}_{1-}$ ${ }_{x} G_{x}$ alloys: A Monte-Carlo simulation with the Stillinger-Weber potential," Phys. Rev. B, vol. 51, no. 8, pp. 4894-4902, Feb. 1995.

[27] J.F. Ziegler, J.P. Biersack and U.Littmark, The Stopping Range of Ions in Solids, vol. 1, Pergamon Press, N.Y., 1983.

[28] N. Chen et al., "Atomic-Scale Simulation for Pseudometallic Defect Generation Kinetics and Effective NIEL in GaN," IEEE Trans. Nucl. Sci., vol. 65 , no. 5, pp. 1108-1118, May 2018.

[29] H. Hensel and H. M. Urbassek, "Implantation and damage under lowenergy Si self-bombardment," Phys. Rev. B, vol. 57, no. 8, pp. 4756-4763, Feb. 1998.

[30] M. S. Janson et al., "Ion implantation range distributions in silicon carbide," J. App. Phys., vol. 93, no. 11, pp. 8903-8909, July 2003.

[31] K. Nordlund et al., "Effect of the interatomic Si-Si potential on vacancy production during ion implantation of Si," Phys. Scr., vol. T54, pp. 34-37, Jan. 1994.

[32] A. Kuronen et al., "First-principles simulation of collision cascades in $\mathrm{S}$ to test pair-potentials for $\mathrm{Si}-\mathrm{Si}$ interaction at $10 \mathrm{eV}-5 \mathrm{keV}, "$ Nucl. Instrum. and Methods Phys. Res. B, vol. 88, no. 4, pp. 382-386, June 1994.

[33] P. Lopez et al., "Molecular dynamics simulations of damage production by thermal spikes in Ge," J. App. Phys., vol. 111, no. 3, p. 033519, Feb. 2012.

[34] K. Nordlund, N. Runeberg and D. Sundholm, "Repulsive interatomic potentials calculated using Hartree-Fock and density-functional theory methods," Nucl. Instrum. and Methods Phys. Res. B, vol. 132, no. 1, pp. 4554, Oct. 1997. 